\title{
Oral presentations
}

\section{RESULTS}

Thirty-two cases were included. Females were overrepresented $(75 \% ; 24 / 32)$. Median age at presentation was 9 years (range $4-13$ ). Three cases (9.4\%) presented with one clinical sign only, and 29 (90.6\%) presented with a combination. The most common clinical sign was a change in mentation $(59.3 \% ; 19 / 32)$, further split into obtundation (84.2\%; 16/19) and changes in behaviour (42.1\%; 8/19). The second most common presentation was weakness $(56.3 \% ; 18 / 32)$; which was further split into generalised (72.2\%; 13/18), posterior weakness $(22 \%$; $4 / 18$ ) and exercise intolerance (16.6\%; 3/18). Additional commonly reported signs included ataxia $(34.4 \%$; 11/32) muscle fasciculation $(34.4 \% ; 11 / 32)$, epileptic seizures (31.3\%; 10/32), collapse (25\%; 8/32), polyphagia $(6.3 \%$;
2/32), PUPD (3.1\%, 1/32) and paroxysmal dyskinesia (3.1\%; 1/32). The two most common paired clinical signs were a change in mentation and weakness (37.9; 11/29). The median duration of clinical signs prior to diagnosis was 1.5 months (range 1 day-3 years).

\section{STATEMENT (CONCLUSIONS)}

The results of this study suggest that the clinical presentation of canine insulinoma differs from what has previously been reported, with mentation and behavioural changes being more common than weakness and epileptic seizures. Therefore, better understanding of this clinical presentation could enhance the diagnostic work up of these patients and lead to earlier tumour diagnosis.

\section{Lomustine,} methotrexate, and cytarabine chemotherapy as a rescue treatment for feline lymphoma

\section{Katherine Smallwood ${ }^{1}$, Aaron Harper², Laura Blackwood ${ }^{1}$}

1 Institute of Veterinary Science, University of Liverpool, Leahurst Campus, Neston, United Kingdom

2 Southfields Veterinary Specialists, Essex, United Kingdom

\section{OBJECTIVES}

Assess the efficacy and tolerability of lomustine, methotrexate, and cytarabine chemotherapy as rescue for feline lymphoma.

\section{METHODS}

Medical records of fifteen cats treated with lomustine, methotrexate and cytarabine for relapsed high-grade feline lymphoma, at a single institution between 2013 and 2018, were examined. All anatomical types were included. Data were analysed using descriptive statistics.

\section{RESULTS}

Nine cats received all three drugs, four cats received only two drugs due to progressive disease, and two cats intentionally received only two drugs. In cats that received (or in which there was intention to treat with) all three drugs, $6 / 13$ (46\%) demonstrated a response to chemotherapy. The cat that intentionally received only lomustine and cytarabine achieved partial remission. The cat that intentionally received only lomustine and methotrexate experienced progressive disease. Treatment was generally well tolerated, although two cats experienced Veterinary Comparative Oncology Group (VCOG) grade 3 neutropenia and one cat experienced VCOG grade 3 thrombocytopenia. The median progression-free survival (PFS) time was 42 days (range: 16-1246), independent of the inclusion/exclusion of the two cats that intentionally received only two drugs.

\section{STATEMENT (CONCLUSIONS)}

COP and CHOP are established first-line chemotherapy for feline lymphoma, but standard rescue protocols are lacking. Lomustine has become a popular single agent option, but prolonged or cumulative myelosuppression can result in treatment delays risking relapse. Therefore, a multidrug lomustine based protocol may be advantageous, and from first principles, should also better overcome resistance. This study suggests that lomustine, methotrexate and cytarabine may represent an efficacious and well tolerated protocol for feline lymphoma rescue. 